Bull. Austral. Math. Soc.

VoL. 59 (1999) [177-180]

\title{
TWISTED HILBERT SPACES
}

\section{Félix Cabello Sánchez}

\begin{abstract}
A Banach space $X$ is called a twisted sum of the Banach spaces $Y$ and $Z$ if it has a subspace isomorphic to $Y$ such that the corresponding quotient is isomorphic to $Z$. A twisted Hilbert space is a twisted sum of Hilbert spaces. We prove the following tongue-twister: there exists a twisted sum of two subspaces of a twisted Hilbert space that is not isomorphic to a subspace of a twisted Hilbert space. In other words, being a subspace of a twisted Hilbert space is not a three-space property.
\end{abstract}

\section{INTRODUCTION}

A Banach space $X$ is called a twisted sum of the Banach spaces $Y$ and $Z$ if it has a subspace isomorphic to $Y$ whose corresponding quotient is isomorphic to $Z$, or else, if there exists an exact sequence

$$
0 \rightarrow Y \rightarrow X \rightarrow Z \rightarrow 0
$$

where the arrows represent bounded linear operators. This note is about "twisted" Hilbert spaces (that is, twisted sums of Hilbert spaces). We construct a twisted sum of two subspaces of twisted Hilbert spaces that cannot be embedded in a twisted Hilbert space, thus answering in part to a question of Castillo, González and Yost [1, p.95].

\section{THE EXAMPLE}

The example is based on the space $Z_{2}$ of Kalton and Peck [4] whose construction we briefly sketch. Consider the homogeneous map $F: l_{2} \rightarrow l_{2}$ defined as

$$
F\left(\sum_{i=1}^{n} x_{i} e_{i}\right)=\sum_{i=1}^{n}\left(\log \|x\|-\log \left|x_{i}\right|\right) x_{i} e_{i}
$$

It can be proved that for $x, y \in l_{2}$ one has

$$
\|F(x+y)-F x-F y\| \leqslant K(\|x\|+\|y\|)
$$

Received 20th April, 1998

The author was supported in part by DGICYT project PB94-1052-C02-02.

Copyright Clearance Centre, Inc. Serial-fee code: 0004-9729/99 \$A2.00+0.00. 
so that $F$ is quasi-linear. Observe that $F$ is only defined for finitely supported sequences. It can be extended to the whole of $l_{2}$ keeping quasi-linearity ([4], [1, p.90]). The space $Z_{2}$ is $l_{2} \oplus_{F} l_{2}$, that is, the product space $l_{2} \times l_{2}$ equipped with the quasi-norm

$$
\|(y, z)\|_{F}=\|y-F z\|_{2}+\|z\|_{2} .
$$

Actually this is only a quasi-norm, but it is equivalent to a norm by results of Kalton $\left([3],\left[1\right.\right.$, p.19]). Observe that $Z_{2}$ contains an isometric copy of $l_{2}$ (the subspace $\{(y, 0)$ : $\left.y \in l_{2}\right\}$ ) whose corresponding quotient is also isometric to $l_{2}$, so that there is an exact sequence

$$
0 \rightarrow l_{2} \rightarrow Z_{2} \rightarrow l_{2} \rightarrow 0
$$

Kalton and Peck proved that this sequence does not split and therefore $Z_{2}$ is a twisted Hilbert space but not itself a Hilbert space. (An earlier example was given by Enflo, Lindenstrauss and Pisier [2], [1, p.82].)

Consider now the subspace $Z$ of $Z_{2}$ spanned by the sequence $\left\{\left(0, e_{i}\right)\right\}$, where $\left\{e_{i}\right\}$ is the standard basis of $l_{2}$. (This space is isomorphic to the Orlicz sequence space $l_{M}$, being $M(t)=(t \log t)^{2}$, [4, Lemma 5.3] but the following description of $Z$ will simplify the exposition.) We want to see that $\left(0, e_{i}\right)$ is a symmetric basis. That $\left\{\left(0, e_{i}\right)\right\}$ is a basic sequence is an immediate consequence of Nikolskii's criterion since, for $n<m$, one has

$$
\begin{aligned}
\left\|\sum_{i=1}^{n} x_{i}\left(0, e_{i}\right)\right\|_{F} & =\left\|F\left(\sum_{i=1}^{n} x_{i} e_{i}\right)\right\|_{2}+\left\|\sum_{i=1}^{n} x_{i} e_{i}\right\|_{2} \\
& \leqslant\left\|F\left(\sum_{i=1}^{m} x_{i} e_{i}\right)\right\|_{2}+\left\|\sum_{i=1}^{m} x_{i} e_{i}\right\|_{2} \\
& =\left\|\sum_{i=1}^{m} x_{i}\left(0, e_{i}\right)\right\|_{F}
\end{aligned}
$$

Moreover, for every permutation $\pi$ of the integers and every choice of signs $\sigma_{i}= \pm 1$, one has

$$
\left\|\sum_{i=1}^{n} x_{i}\left(0, e_{i}\right)\right\|_{F}=\left\|\sum_{i=1}^{n} \sigma_{i} x_{i}\left(0, e_{\pi(i)}\right)\right\|_{F}
$$

since $l_{2}$ has symmetric norm:

$$
\left\|\sum_{i=1}^{n} x_{i} e_{i}\right\|_{2}=\left\|\sum_{i=1}^{n} \sigma_{i} x_{i} e_{\pi(i)}\right\|_{2}
$$

and also

$$
\left\|F\left(\sum_{i=1}^{n} x_{i} e_{i}\right)\right\|_{2}=\left\|F\left(\sum_{i=1}^{n} \sigma_{i} x_{i} e_{\pi(i)}\right)\right\|_{2}
$$


Hence $\left\{\left(0, e_{i}\right)\right\}$ is a symmetric basis (even with symmetric norm). We identify $Z$ with a sequence space via the basis which we denote by $\left\{\nu_{n}\right\}$ (instead of $\left\{\left(0, e_{n}\right)\right\}$ ). The (quasi)-norm of $Z$ will be denoted by $\|\cdot\|_{Z}$. It is not hard to verify that $Z$ satisfies the following three conditions:

(a) $\left\|\nu_{n}\right\|_{Z}=1$ for all $n$;

(b) $\|z\|_{\infty} \leqslant C\|z\|_{Z}$ for some $C$ and all $z \in Z$;

(c) $\|s z\|_{Z} \leqslant M\|s\|_{\infty}\|z\|_{Z}$ for some $M$ and all $s \in l_{\infty}, z \in Z$,

so that the method of [4] still works. Define for $z=\sum_{i=1}^{n} z_{i} \nu_{i}$

$$
G(z)=\sum_{i=1}^{n}\left(\log \|z\|_{Z}-\log \left|z_{i}\right|\right) z_{i} \nu_{i}
$$

Then $G$ is quasi-linear on the finitely supported sequences of $Z$ and can be extended to a quasi-linear map $G: Z \rightarrow Z$. Let $Z \oplus_{G} Z$ be the twisted sum induced by $G$, that is, the algebraic product space $Z \times Z$ endowed with the quasi-norm

$$
\|(y, z)\|_{B}=\|y-G z\|_{Z}+\|z\|_{Z}
$$

which is always equivalent to a norm.

ClaIM. The space $Z \oplus_{G} Z$ cannot be embedded in a twisted Hilbert space.

ProOf: Let us estimate the cotype 2 constants which are the least numbers $a_{n, 2}$ such that, for $x_{1}, \ldots, x_{n}$ in $Z \oplus_{G} Z$,

$$
\left[\int_{0}^{1}\left\|\sum_{i=1}^{n} r_{i}(t) x_{i}\right\|^{2} d t\right]^{1 / 2} \leqslant a_{n, 2}\left[\sum_{i=1}^{n}\left\|x_{i}\right\|^{2}\right]^{1 / 2}
$$

where $r_{i}$ is the sequence of Rademacher functions. Take $x_{i}=\left(0, \nu_{i}\right)$. Then

$$
\sum_{i=1}^{n}\left\|x_{i}\right\|_{G}^{2}=n
$$


while

$$
\begin{aligned}
\int_{0}^{1}\left\|\sum_{i=1}^{n} r_{i}(t) x_{i}\right\|_{G}^{2} d t & =\int_{0}^{1}\left\|\left(0, \sum_{i=1}^{n} r_{i}(t) \nu_{i}\right)\right\|_{G}^{2} d t \\
& \geqslant \int_{0}^{1}\left\|G\left(\sum_{i=1}^{n} r_{i}(t) \nu_{i}\right)\right\|_{Z}^{2} d t \\
& =\int_{0}^{1}\left\|\sum_{i=1}^{n}\left\{\log \left\|\sum_{j=1}^{n} r_{j}(t) \nu_{j}\right\|_{Z}\right\} r_{i}(t) \nu_{i}\right\|_{Z}^{2} d t \\
& =\int_{0}^{1}\left[\left\|\sum_{i=1}^{n} r_{i}(t) \nu_{i}\right\|_{Z} \log \left\|\sum_{j=1}^{n} r_{j}(t) \nu_{j}\right\|_{Z}\right]^{2} d t \\
& =\left[\left\|\sum_{i=1}^{n} \nu_{i}\right\| z \log \left\|\sum_{i=1}^{n} \nu_{i}\right\|_{Z}\right]^{2} \\
& \geqslant \frac{1}{16} n \log ^{4}(n),
\end{aligned}
$$

since a straightforward computation shows that $\left\|\sum_{i=1}^{n} \nu_{i}\right\|_{Z} \geqslant\left(n^{1 / 2} \log n\right) / 2$. This obviously implies that $a_{n, 2}\left(Z \oplus_{G} Z\right) \geqslant c \log ^{2} n$. But Kalton and Peck proved in [4, Theorem $6.2(\mathrm{a})]$ that for a twisted Hilbert space $T$ one has $a_{n, 2}(T) \leqslant C \log n$. Hence $Z \oplus_{G} Z$ is not a subspace of a twisted Hilbert space and the proof is complete.

\section{REFERENCES}

[1] J.M.F. Castillo and M. Gonzáles, Three-space problems in Banach space theory, Lecture Notes in Mathematics 1667 (Springer Verlag, Berlin, Heidelberg, New York, 1997).

[2] P. Enflo, J. Lindenstrauss and G. Pisier, 'On the "three-space" problem', Math. Scand. 36 (1975), 199-210.

[3] N.J. Kalton, 'The three space problem for locally bounded $F$-spaces', Compositio Math. 37 (1978), 243-276.

[4] N.J. Kalton and N.T. Peck, 'Twisted sums of sequence spaces and the three space problem', Trans. Amer. Math. Soc. 255 (1979), 1-30.

Departamento de Matemáticas

Universidad de Extremadura

Avda de Elvas $s / n$

06071 Badajoz

Spain

e-mail: fcabello@unex.es 